\title{
High-Resolution MRI Findings following Trigeminal Rhizotomy
}

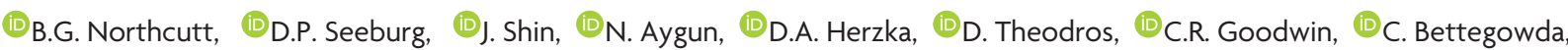
(1DM. Lim, and (1DA.M. Blitz

\begin{abstract}
BACKGROUND AND PURPOSE: Patients with trigeminal neuralgia often undergo trigeminal rhizotomy via radiofrequency thermocoagulation or glycerol injection for treatment of symptoms. To date, radiologic changes in patients with trigeminal neuralgia post-rhizotomy have not been described, to our knowledge. The aim of this study was to evaluate patients after trigeminal rhizotomy to characterize post-rhizotomy changes on 3D high-resolution MR imaging.
\end{abstract}

MATERIALS AND METHODS: A retrospective review of trigeminal neuralgia protocol studies was performed in 26 patients after rhizotomy compared with 54 treatment-naïve subjects with trigeminal neuralgia. Examinations were reviewed independently by 2 neuroradiologists blinded to the side of symptoms and treatment history. The symmetry of Meckel's cave on constructive interference in steadystate and the presence of contrast enhancement within the trigeminal nerves on volumetric interpolated breath-hold examination images were assessed subjectively. The signal intensity of Meckel's cave was measured on coronal noncontrast constructive interference in steady-state imaging on each side.

RESULTS: Post-rhizotomy changes included subjective clumping of nerve roots and/or decreased constructive interference in steadystate signal intensity within Meckel's cave, which was identified in 17/26 (65\%) patients after rhizotomy and 3/54 (6\%) treatment-naïve patients $(P<.001)$. Constructive interference in steady-state signal intensity within Meckel's cave was, on average, $13 \%$ lower on the side of the rhizotomy in patients posttreatment compared with a $1 \%$ difference in controls $(P<.001)$. Small regions of temporal encephalomalacia were noted in $8 / 26(31 \%)$ patients after rhizotomy and $0 / 54(0 \%)$ treatment-naïve patients $(P<.001)$.

CONCLUSIONS: Post-trigeminal rhizotomy findings frequently include nerve clumping and decreased constructive interference in steady-state signal intensity in Meckel's cave. Small areas of temporal lobe encephalomalacia are encountered less frequently.

ABBREVIATIONS: CISS = constructive interference in steady-state; $\mathrm{SI}=$ signal intensity; VIBE = volumetric interpolated breath-hold examination; SPACE $=$ sampling perfection with application-optimized contrasts by using different flip angle evolution

$\mathbf{T}$ rigeminal neuralgia is a debilitating condition characterized by sharp pain in the distribution of the trigeminal nerve. First described in 1773 by John Fothergill, trigeminal neuralgia is now a widely recognized and frequently encountered condition with a prevalence as high as 200/100,000 individuals and an overall incidence of 2.7/100,000/year. ${ }^{1-5}$ First-line treatment commonly consists of medical management with carbamazepine, followed by additional second- and third-line medical treatments. ${ }^{1,6}$ If medical management fails, imaging of the trigeminal nerves is often

Received January 21, 2016; accepted after revision April 16.

From the Departments of Radiology and Radiologic Sciences, Division of Neuroradiology (B.G.N., D.P.S., J.S., N.A., A.M.B.), Biomedical Engineering (D.A.H.), and Neurosurgery (D.T., C.R.G., C.B., M.L.), Johns Hopkins Hospital, Baltimore, Maryland.

Please address correspondence to Ari M. Blitz, MD, Johns Hopkins Medical Institutions, Diagnostic Radiology, Division of Neuroradiology, 600 North Wolfe St, Baltimore, MD 21287; e-mail: ablitz1@jhmi.edu

http://dx.doi.org/10.3174/ajnr.A4868 performed to assess causes such as compression of the cisternal segment of the trigeminal nerve from vascular structures or, less commonly, mass lesions along the course of this nerve.

Trigeminal rhizotomy, which is performed by percutaneous insertion of a needle through the foramen ovale into Meckel's cave to damage the nerve by balloon compression, glycerol injection, or radiofrequency thermocoagulation, is often performed as a first-line procedure and may be the only procedure available to patients unable to undergo the more invasive surgical intervention of microvascular decompression. Microvascular decompression is an invasive method of treatment with reported higher patient satisfaction and an overall lower symptom recurrence rate compared with rhizotomy, but it requires an open neurosurgical approach.,

High-resolution MR imaging of the trigeminal nerves has allowed radiologists to see the cisternal and Meckel's cave segments of the trigeminal nerve with exquisite detail. In particular, con- 
structive interference in steady-state (CISS) imaging, a free precession technique with intrinsic flow suppression and high signalto-noise ratio, allows visualization of fine structures, including individual rootlets of the trigeminal nerve in Meckel's cave. We have also observed that CISS is sensitive to small perturbations in the content of fluid and can demonstrate reduced signal compared with CSF, even when differences are not visualized on spinecho-based imaging. Because patients can have high-resolution imaging after trigeminal rhizotomy for a number of indications,

Table 1: Subject age and sex

Post-Rhizotomy Treatment-Naïve $P$ Value

\begin{tabular}{lccc}
\hline No. of Subjects & 26 & 54 & \\
Age (mean) (range) (yr) & $60(27-85)$ & $55(29-74)$ & .09 \\
Sex & 6 Men, & 20 Men, & .31 \\
& 20 women & 34 women & \\
\hline
\end{tabular}

Table 2: Frequency of findings within Meckel's cave in treatmentnaïve versus post-rhizotomy patients ${ }^{\mathrm{a}}$

\begin{tabular}{lccc}
\hline & \multicolumn{3}{c}{ Treatment- } \\
& Post-Rhizotomy & Naïve & P Value \\
\hline Subjective clumping & $16 / 26(62 \%)$ & $3 / 54(6 \%)$ & $<.001$ \\
Decreased CISS & $13 / 26(50 \%)$ & $3 / 54(6 \%)$ & $<.001$ \\
Subjective clumping without & $4 / 26(15 \%)$ & $0 / 54(0 \%)$ & .01 \\
$\quad$ decreased CISS SI & & & \\
$\downarrow$ CISS SI without clumping & $1 / 26(4 \%)$ & $0 / 54(0 \%)$ & .33 \\
$\begin{array}{l}\text { Subjective nerve clumping } \\
\text { and } \downarrow \text { CISS SI }\end{array}$ & $12 / 26(46 \%)$ & $3 / 54(6 \%)$ & $<.001$ \\
$\begin{array}{l}\text { Subjective nerve clumping } \\
\text { and/or } \downarrow \text { CISS SI }\end{array}$ & $17 / 26(65 \%)$ & $3 / 54(6 \%)$ & $<.001$ \\
\hline
\end{tabular}

Note:- $\downarrow$ indicates decrease.

${ }^{a}$ Interobserver agreement $=90 \%, \kappa=0.69$.

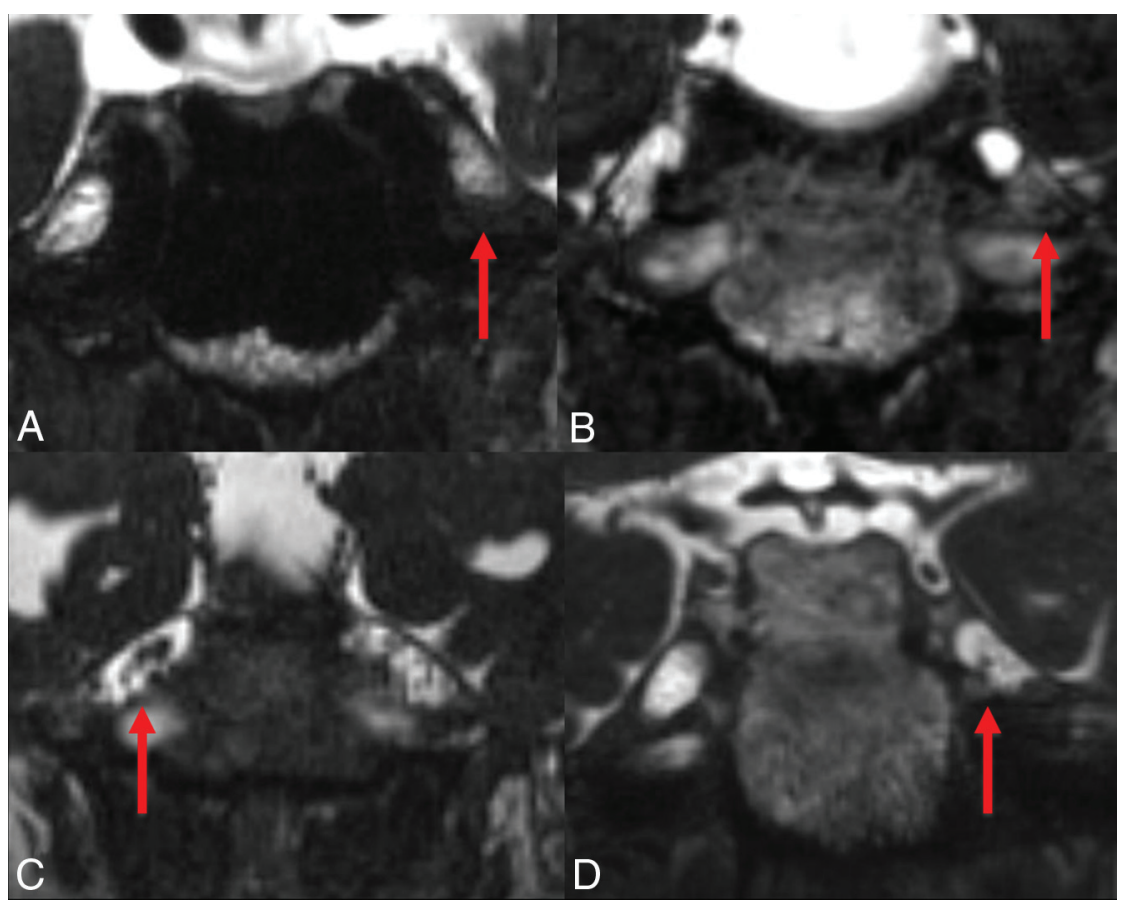

FIG 1. Coronal CISS precontrast images at the level of Meckel's cave. A, Decreased T2 signal intensity and poorly delineated nerve rootlets in the left Meckel's cave after rhizotomy. Note a normal-appearing right Meckel's cave. B, A different patient with clumping of the nerve rootlets inferiorly within the left Meckel's cave post-rhizotomy. C, Central clumping of nerve rootlets in the right Meckel's cave post-rhizotomy. D, A different patient with more subtle clumping of the nerve rootlets in the left Meckel's cave and subtle decreased CISS SI post-rhizotomy. including recurrence of symptoms, the goal of this study was to determine the findings expected on postprocedural imaging following percutaneous rhizotomy.

Given the postulated mechanism of action of rhizotomy - that is, changes in osmolarity and resulting demyelination and neurolysis with glycerol or direct heat neurolysis with radiofrequency thermocoagulation - we hypothesized the following: the rootlets of the trigeminal nerve in the region of the injection would demonstrate visible changes in their course due to clumping and adhesion; and the signal on CISS imaging would be reduced within Meckel's cave due to injectate and/or inflammatory debris. ${ }^{9}$ In addition, we sought to evaluate whether enhancement of the trigeminal nerve or Meckel's cave should be expected following rhizotomy. The rates of other changes to surrounding structures and the muscles of mastication that are innervated by the mandibular division of the trigeminal nerve (cranial nerve V.3) were recorded.

\section{MATERIALS AND METHODS \\ Patient Sample}

A retrospective review of dedicated high-resolution 3D trigeminal neuralgia protocol MR imaging studies was performed from 2011 to 2014. The study was approved by the institutional review board and was Health Insurance Portability and Accountability Actcompliant. Three hundred ten examinations were performed on 261 patients during the study period. Subjects were stratified into preprocedural trigeminal neuralgia and post-rhizotomy groups. Subjects were included in the post-rhizotomy group if they had a documented history of either glycerol and/or radiofrequency thermocoagulation rhizotomy before imaging. All rhizotomies were performed with fluoroscopic image guidance. Subjects were excluded from both groups if they had symptoms atypical for trigeminal neuralgia $(n=51)$, mass lesions in Meckel's cave $(n=11)$, and a history of microvascular decompression $(n=65)$ or gamma knife treatment $(n=4)$ before imaging. Twenty-six studies qualified for the post-rhizotomy group. One hundred fifty-three examinations qualified for the preprocedural trigeminal neuralgia control group, of which 54 were selected at random at approximately 2 controls per case with no statistically significant difference between age and sex compared with the post-rhizotomy group.

\section{Imaging Technique}

All studies were conducted at our institution on Verio or Trio 3T scanners (75 examinations) or Magnetom Espree or Avanto 1.5T scanners (3 examinations) (Siemens, Erlangen, Germany) by using a high-resolution trigeminal neuralgia protocol. The protocol consisted of a sagittal T1, isovolumetric T2 sampling perfection with application-optimized contrasts by using different flip angle 
evolution sequence (SPACE; Siemens), FLAIR, and axial diffusion-weighted images of the brain. High-resolution sequences were acquired, including CISS pre- and postcontrast (section thickness, $0.6 \mathrm{~mm}$; matrix, 256/256; FOV, $16.9 \times 24.6)$ and volumetric interpolated breath-hold examination (VIBE) pre- and postcontrast (section thickness, $0.8 \mathrm{~mm}$; matrix, 256/256; FOV, $16.9 \times 24.6$ ), with fat saturation applied on the postcontrast VIBE imaging. Postcontrast images were acquired after administration of $0.1 \mathrm{~mL} / \mathrm{kg}$ of gadobutrol (Gadavist; Bayer Schering Pharma, Berlin, German) if the glomerular filtration rate was above 60, and half-dose of gadobenate dimeglumine (MultiHance; Bracco Diagnostics, Princeton, New Jersey) was used if the glomerular filtration rate was below 60 , per institutional protocol. 3D-time-offlight MRA of the circle of Willis was also performed as part of the high-resolution trigeminal neuralgia protocol.

\section{Image and Data Analysis}

The selected treatment-naïve and post-rhizotomy studies were intermixed and reviewed independently by 2 neuroradiologists (with $>5$ years experience) blinded to a history of prior treatment. All study sequences were reviewed on a PACS. Meckel's caves were evaluated subjectively for asymmetry, including clumping of the nerve roots and altered signal intensity on both non-contrast-enhanced and contrast-enhanced CISS sequences. Trigeminal nerves were assessed for enhancement on postcontrast VIBE images. The signal intensity of Meckel's cave was ob-

Table 3: Ratio of CISS SI in treatment-naïve versus postrhizotomy patients

\begin{tabular}{|c|c|c|c|}
\hline & $\begin{array}{l}\text { Control Right } \\
\mathrm{MC} / \text { Left } \\
\mathrm{MC} \text { (mean } \\
\text { ratio } \pm \mathrm{SD} \text { ) }\end{array}$ & $\begin{array}{c}\text { Rhizotomy MC/ } \\
\text { Contralateral } \\
\text { MC (mean } \\
\text { ratio } \pm \text { SD) }\end{array}$ & $P$ Value \\
\hline Ratio of CISS SI & $0.99( \pm 0.09)$ & $0.87( \pm 0.15)$ & $<.001$ \\
\hline
\end{tabular}

Note:-MC indicates Meckel cave.

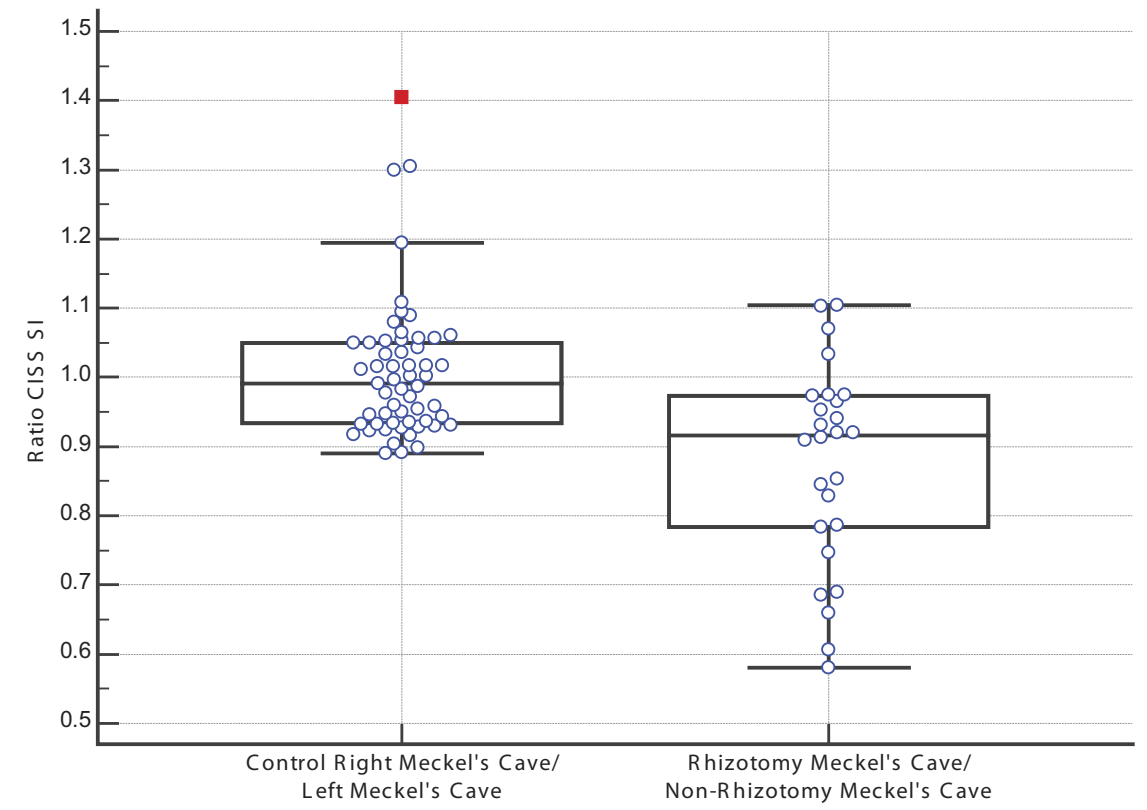

FIG 2. Graph showing the ratio of CISS SI of the right and left Meckel's caves in control patients and rhizotomy/nonrhizotomy in Meckel's caves in post-rhizotomy patients. The control group ratio was 0.99 compared with 0.87 for patients who underwent rhizotomy $(P<.001)$. jectively measured by a central freehand ROI within the largest area of Meckel's cave on coronal non-contrast-enhanced CISS imaging for each case, avoiding Meckel's cave borders. Postprocedural changes in the adjacent structures were evaluated on pre- and postcontrast CISS and VIBE sequences. These adjacent structures included cranial nerves III and VI as they are readily visualized; however, cranial nerve IV was not assessed as it is not well-visualized routinely. Additionally, the medial temporal lobes were assessed as they closely approximate and often abut the lateral dural margin of the Meckel's cave and can be penetrated by piercing the lateral dura of Meckel's cave. ${ }^{10,11}$ The adjacent petrous and cavernous segments of the internal carotid arteries and cavernous sinuses were also assessed on 3D TOF MRA.

\section{Statistical Analysis}

Patients in the control and post-rhizotomy groups were compared for age by using a $t$ test of independent samples, assuming unequal variances, and compared for sex with the Fisher exact test with MedCalc for Windows, Version 15.8 (MedCalc Software; Mariakerke, Belgium). A $P$ value $<.05$ was considered significant.

The sensitivity, specificity, and positive and negative predictive values of subjective nerve clumping or decreased CISS signal intensity (SI) for post-rhizotomy were calculated. Statistical significance was calculated via the Fisher exact test. Interobserver agreement and $\kappa$ were calculated between observers.

A $t$ test of independent samples assuming unequal variance was performed to compare measurements of CISS SI between groups on the basis of the ROI SI values. The Fisher exact test and interobserver agreement were calculated for changes of the structures adjacent to Meckel's cave, including hematoma, encephalomalacia, atrophy of muscles of mastication, cranial nerve III, V, and VI enhancement, and vascular injury of the adjacent petrous and cavernous internal carotid artery, or cavernous-carotid fistula. A $P$ value $<.05$ was considered significant.

\section{RESULTS}

Twenty-six unique subjects were included in the post-rhizotomy group, 12 with glycerol treatment and 14 receiving both glycerol rhizotomy and radiofrequency thermocoagulation rhizotomy before imaging. Fifty-four subjects were included in the trigeminal neuralgia procedure-naïve group. There were no statistically significant differences in age or sex between groups (Table 1).

Subjective nerve clumping or decreased CISS SI was present in 17/26 (65\%) patients after rhizotomy, compared with only $3 / 54(6 \%)$ treatment-naïve patients $(P<.001)$ (Table 2 and Fig 1). Of the 17 patients with changes in Meckel's cave after rhizotomy, 12/17 (71\%) had a 
combination of clumping and decreased overall subjective CISS signal intensity, 4/17 (24\%) demonstrated only subjective clumping of nerve rootlets, and 1/17 (5\%) demonstrated only decreased subjective CISS signal intensity. All 3 treatment-naïve patients with subjective changes in Meckel's cave demonstrated both clumping and decreased CISS signal. No nerve clumping or altered signal was noted in Meckel's cave on VIBE precontrast and postcontrast sequences or FLAIR sequences, though clumping was identified in 2 patients after rhizotomy on T2 SPACE sequences by both reviewers.

In the patients having undergone rhizotomy, objective measurements of CISS SI by ROI in Meckel's cave demonstrated a statistically significant decrease in CISS SI on the side of the rhizotomy compared with the contralateral side and with control groups, with an average $13 \%$ decreased CISS SI on the side of the rhizotomy (Table 3 and Fig 2). Encephalomalacia of the adjacent temporal lobe was present in 8/26 (31\%) patients post-rhizotomy compared with $0 / 54(0 \%)$ patients in the treatment-naïve group $(P<.001)$ (Fig 3). One small hematoma was present in the postrhizotomy group $(4 \%)$, with no hematoma present in the treatment-naïve group $(P=.33)$. All cases of noted encephalomalacia and hematoma on CISS were also identified on FLAIR, VIBE, and T2 SPACE sequences by both reviewers. Atrophy of the muscles of mastication was present in both groups, $3 / 26(12 \%)$ in the rhizotomy group and $3 / 54(6 \%)$ in the treatment-naïve group $(P=$ .38). Cranial nerve enhancement (of CN III, V, and VI) was not present in either group, nor was there damage to the adjacent petrous or cavernous internal carotid arteries or evidence of cavernous carotid fistula $(P=1)$ (Table 4$)$.

The average time of imaging after the most recent rhizotomy was 17.2 months, with a range of 1-63 months. The average time of follow-up of patients with nerve clumping or

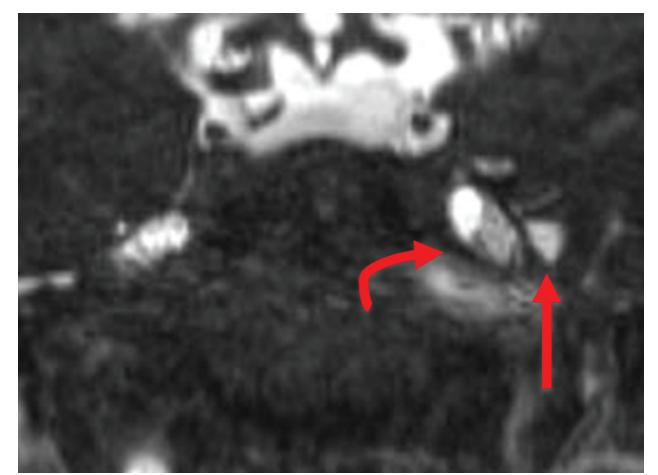

FIG 3. Coronal CISS precontrast at the level of Meckel's caves. Encephalomalacia of the medial left temporal lobe (straight arrow) adjacent to Meckel's cave. Also note clumping of the nerve rootlets in the left Meckel's cave (curved arrow) status post rhizotomy.

Table 4: Frequency of findings in the adjacent structures in treatment-naïve versus postrhizotomy patients

\begin{tabular}{lccccc}
\hline & & & \multicolumn{2}{c}{ Interobserver } \\
& Post-Rhizotomy & Treatment-Naïve & $\boldsymbol{P}$ Value & Agreement & $\boldsymbol{\kappa}$ \\
\hline Encephalomalacia & $8 / 26(31 \%)$ & $0 / 54(0 \%)$ & $<.001$ & $98.8 \%$ & 0.93 \\
Hematoma & $1 / 26(4 \%)$ & $0 / 54(0 \%)$ & .33 & $100 \%$ & 1 \\
Atrophy of muscles of & $3 / 26(10 \%)$ & $3 / 54(4 \%)$ & .38 & $96.3 \%$ & 0.64 \\
$\quad$ mastication & & & & & 1 \\
CN V enhancement & $0 / 26(0 \%)$ & $0 / 54(0 \%)$ & 1.00 & $100 \%$ & 1 \\
Vascular injury & $0 / 26(0 \%)$ & $0 / 54(0 \%)$ & 1.00 & $100 \%$ & 1 \\
\hline
\end{tabular}

Note:- $\mathrm{CN}$ indicates cranial nerve. decreased CISS signal was 16.5 months, compared with 18.4 months in those patients without clumping or CISS signal change $(P=.56)$. The average number of rhizotomy treatments in patients with subjective clumping or decreased CISS SI was 2.9, compared with 1.3 treatments for those who did not have clumping or decreased CISS SI $(P<.01)$. Seven of 12 $(58 \%)$ patients who had undergone rhizotomy with only glycerol treatment had subjective changes in Meckel's cave, while $10 / 14(63 \%)$ patients who had glycerol and radiofrequency treatment had subjective changes $(P=.68)$. All subjects in our sample treated with radiofrequency rhizotomy had also undergone glycerol rhizotomy. The average number of rhizotomies in those with encephalomalacia was 3.8, compared with 1.5 for those without encephalomalacia $(P=.03)$. Three of $12(25 \%)$ patients who had only glycerol treatment had encephalomalacia compared with 6/14 (42\%) patients who had a combination of glycerol and radiofrequency treatment $(P=.43)$.

\section{DISCUSSION}

Studies to date on patients post-rhizotomy have primarily focused on clinical outcomes, notably comparison of different forms of rhizotomy with each other and with microvascular decompression. While these articles offer occasional images of complications associated with rhizotomy, no studies to date, to the authors' knowledge, have evaluated patients after rhizotomy in an effort to describe postprocedural findings on MR imaging. This study was performed to determine what findings can be encountered on postprocedural MR imaging.

Subjective changes, such as decreased CISS SI and clumping of nerve roots, are commonly encountered (65\%) after rhizotomy; however, these changes are rarely seen in treatmentnaïve patients $(6 \%)$. These results suggest that the radiologic findings are associated with the rhizotomy procedure rather than the underlying pathophysiology of trigeminal neuralgia. Objective measurements of signal intensity in Meckel's cave support our subjective findings by showing a statistically significant decrease in CISS SI in Meckel's cave after rhizotomy. The mechanism underlying these changes is presumed to be the result of chemical- or heat-induced neurolysis of the nerve rootlets in Meckel's cave. Notably, the patients with changes in Meckel's cave had undergone, on average, more rhizotomy treatments $(n=2.9)$ compared with those who did not have such changes $(n=1.3)$. Although the cause is unknown, similar changes demonstrated in procedure-naïve patients could reflect a prior inflammatory event predating the development of trigeminal neuralgia. Conversely, there could be recall bias in our study, with the possibility that some subjects failed to report their rhizotomy at another institution to the clinician.

Small foci of encephalomalacia of the temporal lobe adjacent to Meckel's cave were encountered in $31 \%$ of patients postrhizotomy and not in the control group. One such focus demonstrated minimal blood products. These findings could be due to the adjacent temporal lobe being within the thermal zone of the radiofrequency probe and/or direct surgical ma- 
nipulation. ${ }^{10,11}$ No adverse clinical signs or symptoms related to the temporal lobe encephalomalacia were reported in these patients.

Weakness of muscles of mastication frequently occurs after trigeminal rhizotomy, with a recent review noting weakness in $16 \%$ of patients, which is often transient lasting 6-12 months. ${ }^{12}$ In our review, atrophy of the muscles of mastication was present in patients with and without rhizotomy, with no statistically significant difference between groups. No enhancement of trigeminal nerve roots was encountered after treatment, though it is unclear whether more immediate postprocedural imaging would perhaps reveal enhancement in the acute injury phase after treatment. Additional complications such as vascular injury (cavernous carotid fistula, internal carotid artery pseudoaneurysms), abscess, meningitis, and cranial nerve III and VI injury, noted in previous publications, were not encountered clinically or on imaging in our series. ${ }^{10-18}$

There were several limitations in this study, including the inherent biases associated with the retrospective study design. Subjects were grouped according to a review of the medical record, depending on whether the patient was documented as having previously undergone rhizotomy. The patients were seen by experienced clinicians in a dedicated trigeminal neuralgia clinic, and documentation of prior procedures is standard, but we cannot exclude the possibility that subjects had undergone rhizotomy at another institution and failed to report this to the clinician. All patients in our study who had rhizotomy underwent glycerol rhizotomy or glycerol in combination with radiofrequency thermocoagulation rhizotomy. No patients had balloon compression rhizotomy, a method not used at our institution due to the preference of the surgeons. Another limitation of this retrospective study was lack of pre-rhizotomy imaging in those patients who underwent rhizotomy, with the exception of 1 patient. In the case of the 1 patient who underwent high-resolution imaging both before and after treatment, no difference was noted within Meckel's cave or the adjacent structures between studies. Additionally, no correlation between the degree of clumping or decreased CISS SI in Meckel's cave and the degree of symptoms could be made due to the retrospective nature of the study and in the absence of clinically reported grading of symptoms.

The timing of imaging after rhizotomy greatly varied, which could potentially influence the degree of changes seen in Meckel's cave, though the average time of imaging in those with and without nerve clumping was similar. Additionally, more acute changes within Meckel's cave after rhizotomy ( $<1$ month) could not be accurately assessed. Last, the pain of trigeminal neuralgia is known to recur frequently following rhizotomy. Many of the subjects imaged after rhizotomy experienced recurrence of symptoms. Because patients who responded positively to trigeminal rhizotomy are unlikely to have undergone follow-up imaging, whether or to what extent these findings might correlate with degree or duration of therapeutic response remains a subject for further investigation.

\section{CONCLUSIONS}

Post-trigeminal rhizotomy changes in patients with trigeminal neuralgia frequently include nerve clumping and decreased CISS SI within Meckel's cave, findings that are not commonly encountered in patients before treatment. Tiny foci of encephalomalacia can also be seen in the adjacent temporal lobe. Further investigation is necessary to determine whether and how such findings are related to the extent and durability of pain relief following rhizotomy.
Disclosures: C. Rory Goodwin-UNRELATED: Grants/Grants Pending: UNCF Merck Postdoctoral Fellow, * Burroughs Wellcome Fund (award).* Ari M. Blitz-UNRELATED: Payment for Lectures (including service on Speakers Bureaus): Siemens, Comments: honorarium for an educational talk on imaging of the cranial nerves in 2014. Michael Lim—UNRELATED: Consultancy: Bristol-Myers Squibb, Stryker, Comments: research related to my laboratory research in immunotherapy (BMS); Grants/Grants Pending: Stryker, ${ }^{\star}$ Bristol-Myers Squibb, ${ }^{*}$ Aegenus, ${ }^{*}$ Celldex, ${ }^{*}$ Arbor, ${ }^{*}$ ImmunoCellular Therapeutics, ${ }^{\star}$ Comments: grant for studying CSF leak rates in patients after MVD (Stryker), research pertaining to immunotherapy outside the scope of this article (others); Patents (planned, pending or issued): related to immunotherapy*; Travel/Accommodations/ Meeting Expenses Unrelated to Activities Listed: for travel to consultant activities. *Money paid to the institution.

\section{REFERENCES}

1. Prasad S, Galetta S. Trigeminal neuralgia: historical notes and current concepts. Neurologist 2009;15:87-94 CrossRef Medline

2. Penman J, Trigeminal neuralgia. In: Vinken PJ; Bruyn GW, eds. Handbook of Clinical Neurology. Vol 5. Amsterdam: North Holland; 1968:296-322

3. Katusic $S$, Beard CM, Bergstralh E, et al. Incidence and clinical features of trigeminal neuralgia, Rochester, Minnesota, 1945-1984. Ann Neurol 1990;27:89-95 CrossRef Medline

4. Rozen TD. Trigeminal neuralgia and glossopharyngeal neuralgia. Neurol Clin 2004;22:185-206 CrossRef Medline

5. Manzoni GC, Torelli P. Epidemiology of typical and atypical craniofacial neuralgias. Neurol Sci 2005;26(suppl 2):s65-s67 CrossRef Medline

6. Campbell FG, Graham JG, Zilkha KJ. Clinical trial of carbazepine (tegretol) in trigeminal neuralgia. J Neurol Neurosurg Psychiatry 1966;29:265-67 CrossRef Medline

7. Tatli M, Satici O, Kanpolat $Y$, et al. Various surgical modalities for trigeminal neuralgia: literature study of respective long-term outcomes. Acta Neurochir (Wien) 2008;150:243-55 CrossRef Medline

8. Barker FG 2nd, Jannetta PJ, Bisonette DJ, et al. The long-term outcome of microvascular decompression for trigeminal neuralgia. N Engl J Med 1996;334:1077-83 CrossRef Medline

9. Missios S, Mohammadi A, Barnett GH. Percutaneous treatments for trigeminal neuralgia. Neurosurg Clin N Am 2014;25:751-62 CrossRef Medline

10. Arrese I, Lobato R, Alén JF, et al. Acute subdural and intratemporal hematoma as a complication of percutaneous compression of the gasserian ganglion for trigeminal neuralgia. Neurocirugia (Astur) 2005;16:177-82 Medline

11. Rath GP, Dash HH, Bithal PK, et al. Intracranial hemorrhage after percutaneous radiofrequency trigeminal rhizotomy. Pain Pract 2009;9:82-84 CrossRef Medline

12. Tew JM, Morgan CJ, Grande AW. Percutaneous stereotactic rhizotomy in treatment of intractable facial pain. In: Quinones-Hinojosa A, ed. Schmidek and Sweet's Operative Neurosurgical Techniques: Indications, Methods, and Results. 6th ed. Philadelphia: Elsevier Saunders; 2012

13. Taha JM, Tew JM Jr. Comparison of surgical treatments for trigeminal neuralgia: reevaluation of radiofrequency rhizotomy. Neurosurgery 1996;38:865-71 CrossRef Medline

14. Sweet WH, Poletti CE, Roberts JT. Dangerous rises in blood pressure upon heating of trigeminal rootlets: increasing bleeding times in patients with trigeminal neuralgia. Neurosurgery 1985;17:843-44 Medline

15. Sekhar LN, Heros RC, Kerber CW. Carotid-cavernous fistula following percutaneous retrogasserian procedures: report of two cases. J Neurosurg 1979;51:700-06 CrossRef Medline

16. Gökalp HZ, Kanpolat Y, Tümer B. Carotid-cavernous fistula following percutaneous trigeminal ganglion approach. Clin Neurol Neurosurg 1980;82:269-72 CrossRef Medline

17. Harrigan MR, Chandler WF. Abducens nerve palsy after radiofrequency rhizolysis for trigeminal neuralgia: case report. Neurosurgery 1998;43:623-25 Medline

18. Acqui M, Familiari P, Pesce A, et al. Brain abscess after percutaneous therapy for trigeminal neuralgia. Case Rep Infect Dis 2015;2015: 162793 CrossRef Medline 